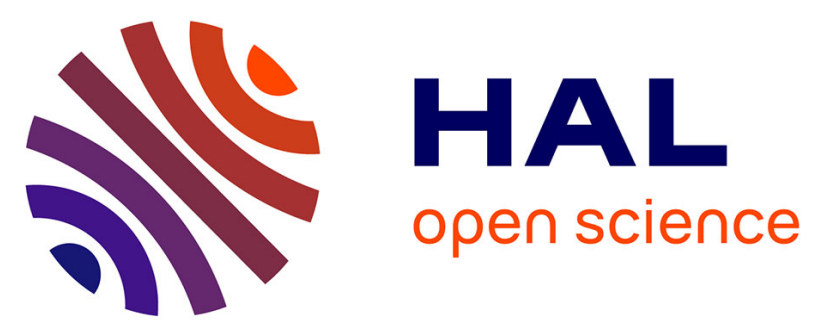

\title{
Performance of a prototype aerogel counter readout by finemesh photo-tubes
}

K. Arisaka, M. Berthet, E. Borsato, D. Boutigny, A. Buzykaev, F. Dalcorso, I. de Bonis, J.M. Dubois, J. Favier, F. Ferroni, et al.

\section{- To cite this version:}

K. Arisaka, M. Berthet, E. Borsato, D. Boutigny, A. Buzykaev, et al.. Performance of a prototype aerogel counter readout by finemesh photo-tubes. Nuclear Instruments and Methods in Physics Research Section A: Accelerators, Spectrometers, Detectors and Associated Equipment, 1997, 385, pp.74-80. in2p3-00014465

\section{HAL Id: in2p3-00014465 https://hal.in2p3.fr/in2p3-00014465}

Submitted on 30 Mar 1999

HAL is a multi-disciplinary open access archive for the deposit and dissemination of scientific research documents, whether they are published or not. The documents may come from teaching and research institutions in France or abroad, or from public or private research centers.
L'archive ouverte pluridisciplinaire HAL, est destinée au dépôt et à la diffusion de documents scientifiques de niveau recherche, publiés ou non, émanant des établissements d'enseignement et de recherche français ou étrangers, des laboratoires publics ou privés. 
K. Arisaka ${ }^{1}$, M. Berthet ${ }^{3}$, E. Borsato ${ }^{2}$, D. Boutigny ${ }^{3}$, A .Buzykaev ${ }^{4}$, F. DalCorso ${ }^{2}$, I. De Bonis ${ }^{3}$, J.M. Dubois ${ }^{3}$, J. Favier ${ }^{3}$, F. Ferroni ${ }^{5}$, M. Foucher ${ }^{6}$, S. Ganzhur ${ }^{4}$, F. Iacovella ${ }^{2}$, H. Jawahery ${ }^{6}$ Y. Karyotakis ${ }^{3}$, G. Kolachev ${ }^{4}$, E. Kravchenko ${ }^{4}$, R. Lafaye ${ }^{3}$, M.A. Mazzoni ${ }^{5}$, V. Mikerov ${ }^{4}$, M. Morandin ${ }^{2}$, S. Morganti ${ }^{5}$, A. Onuchin ${ }^{4}$, J. Oyang $^{7}$, P. Petitpas ${ }^{3}$, G. Piredda ${ }^{5}$, M. Posocco ${ }^{2}$, R. Santacesaria ${ }^{5}$, A. Shamov ${ }^{4}$, R. Stroili ${ }^{2}$, V. Tayursky ${ }^{4}$, V. Telnov ${ }^{4}$, E. Torassa ${ }^{2}$, C. Voci ${ }^{2}$

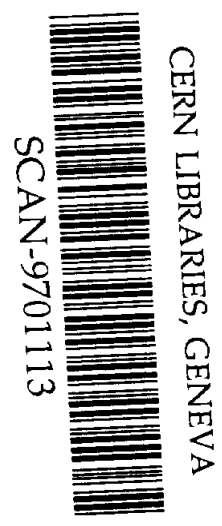

$\operatorname{swg} 704$

\section{Abstract}

The $B A B A R$ experiment, in order to achieve its physics program, requires pion/kaon identification capability up to $4.3 \mathrm{GeV} / \mathrm{c}$. The recent development of new processes has lead to the fabrication of low density silica aerogel with high optical quality. An aerogel threshold counter using the combination of 2 refractive indices (1.055 and 1.007) can be used to complete the angular coverage of the particle identification system in the forward region of the $B A B A R$ experiment.

We present final test-beam results on a 2 layer aerogel prototype, readout by Hamamatsu fine mesh photo-tubes, as required by the high magnetic field environment of the $\mathrm{BaBar}$ experiment. Several configurations have been tested, with different aerogels, photo-tubes and reflective materials.

We have performed a Monte-Carlo simulation, in order to understand the sensitivity of light collection to the optical parameters.

The result of the test shows that such a detector can achieve the desired performances.

\footnotetext{
${ }^{1}$ University of California, Los Angeles, Los Angeles, USA

${ }^{2}$ Università di Padova and INFN, Sezione di Padova, Italy.

${ }^{3}$ L.A.P.P. CNRS/IN2P3 - Annecy-le-Vieux France

${ }^{4}$ Budker Institute of Nuclear Physics, Novosibirsk, Russia

${ }^{5}$ Università di Roma "La Sapienza" and INFN, Sezione di Roma 1, Italy

${ }^{6}$ University of Maryland, College Park, Maryland, USA

${ }^{7}$ California Institute of Technology, Pasadena, California, USA
} 


\section{Introduction}

The main physics goal of the BaBar experiment is the measurement of the $C P$ violation parameters in the neutral $\mathrm{B}$ meson decays. In order to measure the $\alpha$ angle of the unitarity triangle one must be able to distinguish the decay $B^{0} \rightarrow \pi^{+} \pi^{-}$from $B^{0} \rightarrow K^{+} \pi^{-}$. The momentum of these pions or kaons can be as high as $4.5 \mathrm{GeV} / \mathrm{c}$ in the forward region. To measure the time dependent $C P$ violation asymmetry, one should determine the nature of the $\mathrm{B}$ meson emitted in the opposite direction to the one decaying into a $C P$ eigenstate. This tagging can be performed using kaons from the cascade decay $b \rightarrow c \rightarrow s$ In this case, the kaon momentum is mostly below $1.5 \mathrm{GeV} / \mathrm{c}$.

In the BaBar Technical Design Report [1], the particle identification system is completed in the forward direction with an aerogel detector [2][3][4][5][6]. The proposed design is shown in figure 1 . It is a 2 layer, 2 ring geometry permitting $\pi / K$ identification between 0.4 and $4.3 \mathrm{GeV} / \mathrm{c}$. For the inner ring, the first layer is filled with high index aerogel (1.055) read out by 1.5 in. tubes while the second one is filled with low index aerogel (1.007) read out by 2 in. tubes. The indices are reversed for the upper ring. The photo-tubes are connected to the aerogel containers with a pyramidal shaped air light guide. The walls of the containers are wrapped with multiple layers of high reflectivity white PTFE film.

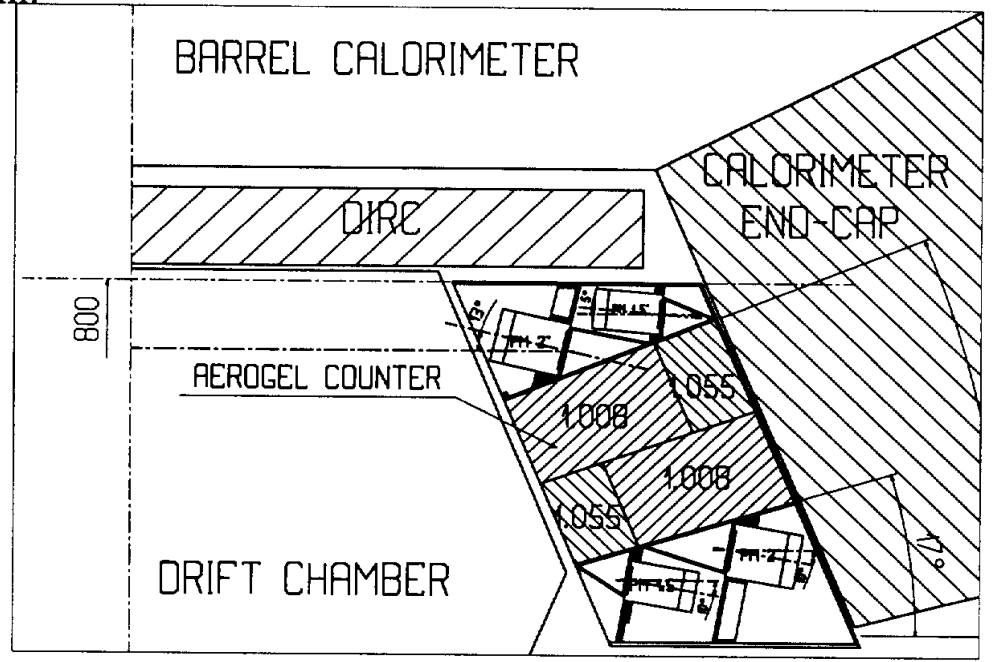

Fig. 1. The BaBar ATC detector in the 2-layer option and fine mesh reading

The photo-tubes used are 19 stage, high quantum efficiency fine mesh from Hamamatsu. They are placed in such a way that the angle between the tube axis and the magnetic field $(1.5 \mathrm{~T})$ direction does not exceed $13^{\circ}$.

In order to test the performances of such a detector, we built a 2 cell prototype, corresponding to one ring, with dimensions close to the mechanical design, 
and with the proposed light guides. This prototype has been tested at the CERN PS T10 test beam together with two other prototypes: one with similar geometry but with hybrid photodiode (HPD) readout [7] and a second one, a 4-layer design, with fine mesh readout [8]. Preliminary results were presented in [9].

\section{Description of the beam line and apparatus}

Beam line description

The T10 beam line can provide positive and negative particle beams with momenta between 1 and $5 \mathrm{GeV} / \mathrm{c}$. The beam composition were mainly pions and protons, with a small kaon and electron contamination.

The beam line (figure 2) was equipped with two $\mathrm{CO}_{2}$ Cherenkov counters, $\mathrm{C} 1$ and $\mathrm{C} 2$, filled to a pressure of 4.3 bar. The beam was defined by the coincidence of three scintillator counters, its extension being limited by the third counter S3 $\left(1 \times 1 \mathrm{~cm}^{2}\right)$. Another thick $(1 \mathrm{~cm})$ veto scintillator counter was read out in a camac scaler, in order to count the number of particles crossing our setup in a $1 \mu \mathrm{sec}$ gate. The three prototypes were placed on a horizontally and vertically moving table.

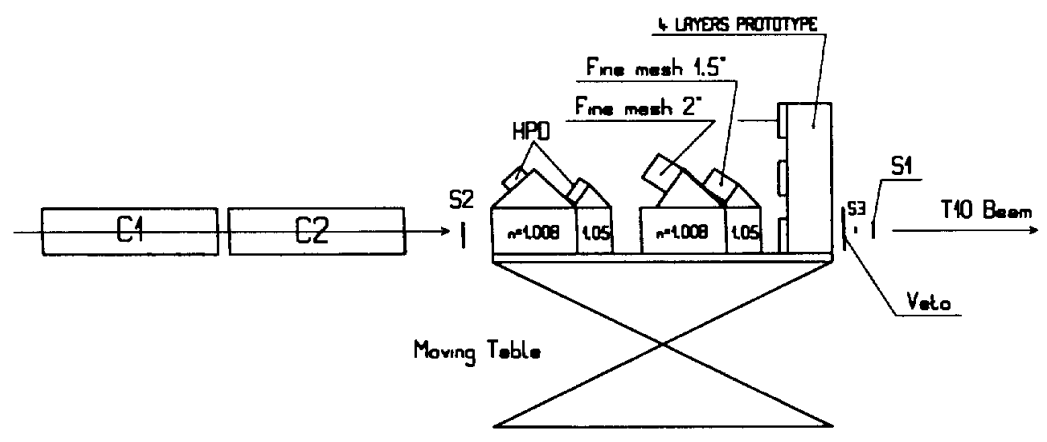

Fig. 2. The T10 beam line with the apparatus

The Trigger

Three kind of triggers were generated :

- Beam. A beam event was the coincidence of the 3 counters S1,S2 and S3 shown in Figure 2 
For some runs, we also included in anti-coincidence the two Cherenkov counters $\mathrm{C} 1$ and $\mathrm{C} 2$ to select protons in the beam.

- Pedestal. We recorded pedestal events inside and outside burst.

- Calibration. The photo-detectors were calibrated using a light emitting diode (LED).

The aerogel prototype counter

As shown in Figure 3, the prototype was composed of 2 separated cells:

- The low index cell, had a dimension of $10 \times 10 \times 14 \mathrm{~cm}^{3}$. It was filled with 5 aerogel slices of $9.5 \times 9.5 \mathrm{~cm}^{2}$ front face and $2.6 \mathrm{~cm}$ thick. The aerogel has been produced by the Jet Propulsion Laboratory (JPL), and had a nominal index of 1.008 . The light was read out with a 2 in., 19 stage, Hamamatsu R5504 fine mesh photomultiplier tube (normal glass window and $26 \%$ quantum efficiency at $400 \mathrm{~nm}$ ). The gain was $\simeq 10^{7}$ at 2300 Volts.

- The high index cell, had a dimension of $10 \times 10 \times 6 \mathrm{~cm}^{3}$. Two sets of aerogel blocks have been tested, one from Airglass with an index $n=1.055$ and another from the Boreskov Catalysis Institute in Novosibirsk [14] with an index $\mathrm{n}=1.050$ (SAN-95). The counter was read out with a 1.5 in., 16 stage Hamamatsu R6148 fine mesh photomultiplier tube (UV window and quantum efficiency $\simeq 20 \%$ at $400 \mathrm{~nm}$ ). The gain was $\simeq 10^{6}$ at 2000 Volts.

We used standard NIM amplifiers with a gain of 5 and 50 for the 2 in. and the 1.5 in. tubes respectively.

The inner walls of the cells were covered by 3 layers of $250 \mu \mathrm{m}$ PTFE film on top of either aluminized mylar or 'Bristol' paper. This configuration has been found to be the best, when compared to the use of milipore 'HAWP- 0.45 ' paper in layers. The air light guides, located on top of each cell, had pyramidal shapes and were wrapped with one layer of aluminized mylar and three layers of $250 \mu \mathrm{m}$ PTFE film. The light guides were covering $100 \%$, and $62 \%$ of the top surface, of the high, and low index cells, respectively (Figure 3).

In order to remove water from the aerogel, all the blocks were baked before exposing them to the beam. The baking procedure was:

- Slow increase by $1.6^{\circ} \mathrm{C} / \mathrm{min}$ from room temperature up to $500^{\circ} \mathrm{C}$.

-3 hours at $500^{\circ} \mathrm{C}$

- Return to room temperature with a maximum decrease slope of $1.6^{\circ} \mathrm{C} / \mathrm{min}$ 


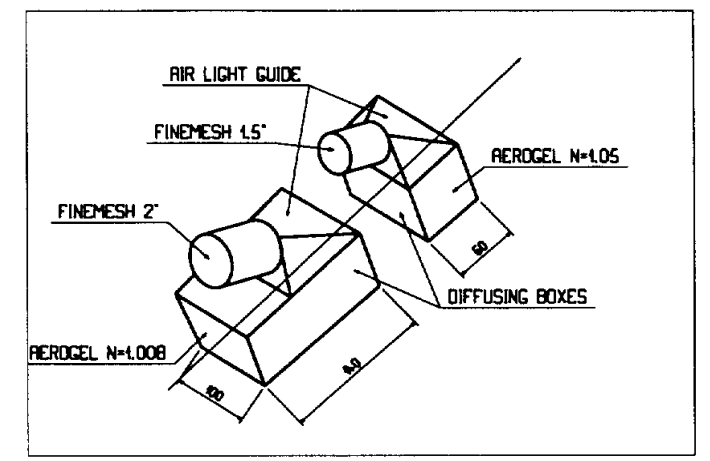

Fig. 3. The prototype counters for low and high index aerogel

\section{One photo-electron calibration}

A fine mesh photo-tube calibration is a delicate procedure as this tube does not show a single photo-electron (p.e.) peak. We injected in the cell, with an optical fiber, the light of an LED tuned such that $95 \%$ of the triggers result in " 0 " p.e. detected (pedestal). A typical spectrum is shown in figure 4.

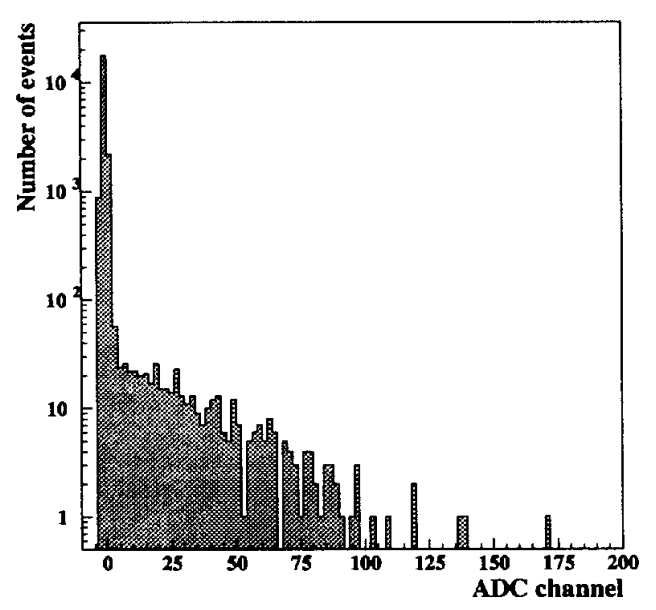

Fig. 4. A LED run for 2" photo tubes. The peak at 0 corresponds to " 0 " photo-electron.

The probability of 1 p.e. appearance is determined by Poisson distribution with an expected value:

$$
\mu=-\ln \frac{n_{0}}{N}
$$

where $n_{0}$ is the number of events contained in the pedestal and $N$ the total number of events.

We fit the shape of the distribution excluding the pedestal, with an exponential or with the tail of a gaussian function. The calibration constant $C$ is then 
computed by the mean value of the fit function between 0 ADC channels and infinity. We assume the validity of the fit function under the pedestal. The exponential fit is good when the number of events outside the pedestal is below $2.5 \%$ but as the ratio $n_{0} / N$ decreases, the shape becomes more and more gaussian.

Contribution from more than 1 p.e. is determined by Poisson distribution. The calibration constant $C$ is then corrected by multiplying it by:

$$
\xi=\frac{1-e^{-\mu}}{\mu}=\frac{\frac{n_{0}}{N}-1}{\ln \frac{n_{0}}{N}}
$$

We estimated the total error for the calibration to be $3.5 \%$ and $5 \%$ for the 2 " and 1.5" tubes respectively. The difference between these errors reflects their pedestal distribution widths.

\section{Results on high index aerogel}

Aerogel cells were exposed to a $5 \mathrm{GeV} / \mathrm{c}$ negative pion beam $(\beta \simeq 1)$. The events were selected by requiring a clean signal in both gas Cherenkov counters, and by asking only 1 count within $1 \mu$ sec in the veto counter to reduce pile up events. Figure 5 (dashed curve) shows the observed number of photoelectrons, for the Airglass $(n=1.055)$ aerogel, as a function of the vertical distance $(y)$ to the center of the box. The light guide entrance was placed on top of the cell at $y=5 \mathrm{~cm}$. The beam was centered in the horizontal plane $(x=0)$. One can observe a strong decrease in the number of photo-electrons with the distance to the light guide entrance. At the center of the cell we measured $6 \pm 0.3$ photo-electrons.

The solid line on figure 5 corresponds to the same scan for a cell filled with the Novosibirsk SAN-95 $(n=1.050)$ aerogel. The number of photo-electrons is larger than for the Airglass one; we observe more than 10 photo-electrons for every beam position. The dependence with the distance to the light guide is less pronounced. This last aerogel shows clearly a better optical quality than the previous one. 


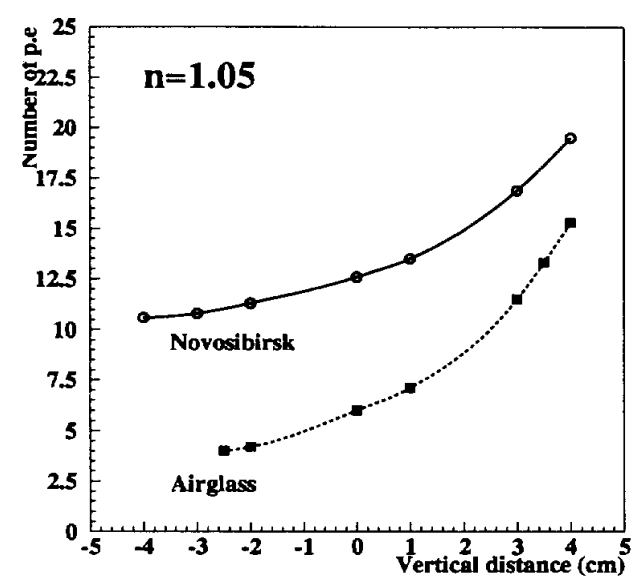

Fig. 5. Number of photo-electrons versus the vertical distance to the center of the box. The photo-multiplier is located at $y=5 \mathrm{~cm}$. The solid line corresponds to the Novosibirsk SAN-95 high index aerogel, and the dashed line corresponds to the Airglass aerogel

\section{Results on low index aerogel}

Response to $\beta \simeq 1$ particles

We have tested a low index aerogel $(n=1.008)$ from JPL, with the same 5 $\mathrm{GeV} / \mathrm{c}$ pion beam $(\beta \simeq 1)$ selected in the same way as described in the previous section. The dashed line in figure 6 shows the result of a scan in the vertical direction. We also observe a variation of the number of detected photo-electrons with the distance to the light guide entrance but weaker than for the high index. Nevertheless, due to the bad single photo-electron response of the finemesh photomultiplier tubes we consider that the absolute number of photo-electrons measured here is too small for safe operation in the BaBar detector.

In order to increase the amount of photo-electrons, we made use of the PMP420 [11] wavelength shifter. We have added one more layer of PTFE on the walls parallel to the beam direction. This layer was impregnated with wavelength shifter dissolved in acetone. The solid line in figure 6 shows the result of the scan in the vertical plane. With this layout we observe a clear increase of the number of photo-electrons and a weaker dependence with the distance to the light collection device. The total increase is $35 \%$ in the center and $65 \%$ at $-3 \mathrm{~cm}$. The wavelength shift from $\simeq 350 \mathrm{~nm}$ to $\simeq 420 \mathrm{~nm}$ has the obvious advantage to decrease both the absorption and the Rayleigh scattering which is responsible for increasing the photon path length. This effect is naturally 


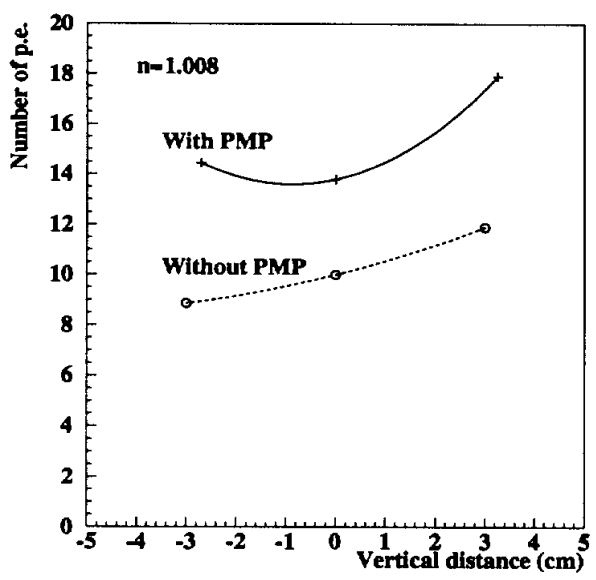

Fig. 6. Number of photo-electrons versus the vertical distance to the center of the box. The dashed line corresponds to $n=1.008$ index aerogel in a cell with a standard PTFE wrapping. Solid line corresponds to the same aerogel block but inside a cell with an added layer of PTFE impregnated with PMP420

enhanced when the crossing particle is close to a shifting wall.

With PMP wavelength shifter, we obtain more than 13 photo-electrons for every position of the incident particles in the cell.

We do not observe a strong dependence of the signal with the position of incident beam in the horizontal plane. A displacement of the impact point by $2 \mathrm{~cm}$ horizontally around the central position, leads to a variation in the average number of photo-electrons smaller than $5 \%$.

We have developed a simple Monte-Carlo simulation of the fine mesh response based on the work described in reference [12]. The inputs of the simulation are: the total gain of the tube $\left(0.9 \times 10^{7}\right)$, the expected number of photo-electrons, the probability for an electron to interact with one mesh plane and a gaussian fluctuation function. A converted photo-electron is amplified through the 19 stages of the tube and the collected electrons are converted to ADC counts. Taking a probability for the electron to be amplified by the meshes equal to $\simeq 30 \%$, one can see on figure 7 that we reproduce the observed spectrum for $\mathrm{N}=13.4$ p.e.

To check the exact value of the aerogel index of refraction, we fitted the pions threshold curve on $1,1.1,1.2$ and $1.5 \mathrm{GeV} / \mathrm{c}$ pion momenta runs. The result is a value of $n=1.0076 \pm 0.0001$ at $500 \mathrm{~nm}$. 


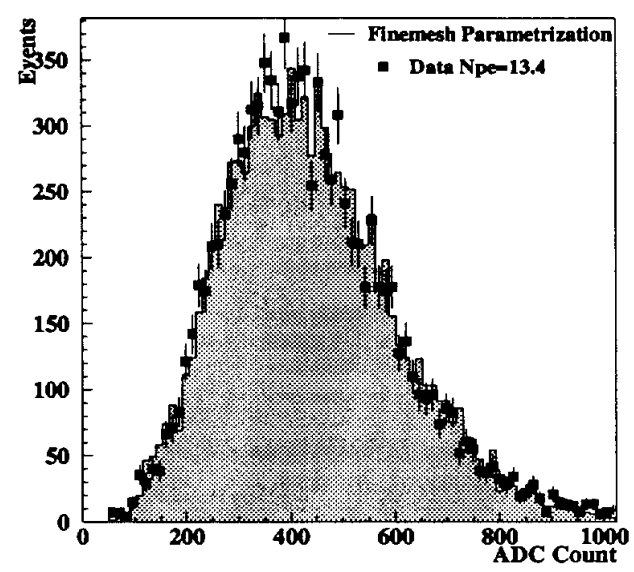

Fig. 7. Measured fine mesh response for pions at $5 \mathrm{GeV} / \mathrm{c}$ corresponding to 13.4 p.e (squares) and comparison with a simple modelization of the fine mesh (solid line). The best agreement between data and Monte-Carlo is obtained for a $30 \%$ probability for an electron to interact with a mesh plane

Study of below threshold particles and background

We have studied the signal induced by below threshold particles. For this purpose, we have used a $5 \mathrm{GeV} / \mathrm{c}$ proton beam selected by requiring the absence of signal in both gas Cherenkov counters and by eliminating events giving more than 1 count in the veto counter. The use of a further $n=1.012$ aerogel counter equipped with a 3 in. tube have permitted to find a kaon contamination in the beam at the level of $\simeq 5 \%$ of the protons. In order to decrease the kaon contamination we have cut the events giving signal in the low index (1.008) prototype read out by HPD, located in front of our counter.

At $5 \mathrm{GeV} / \mathrm{c}$, for a run in which the average number of photo-electrons for $\beta=1$ particle is equal to 13.4 (diffusing box with PMP), we find a background of 0.86 photo-electrons induced by below threshold protons. If we remove events compatible with 0 photo-electrons by setting a very low threshold at $1.5 \sigma$ from the pedestal, we keep $40 \%$ of the events with an average number of photo-electrons equal to 2.2 .

The background is due to $\delta$ rays, secondary interactions of the beam, Cherenkov light in the PTFE wrapping,and scintillation in either aerogel or wrapping. From a detailed GEANT simulation of the beam line materials we find that at $5 \mathrm{GeV} / \mathrm{c}, \simeq 1.5 \%$ of the protons will produce delta rays above the Cherenkov threshold in the aerogel. Most of them (56\%) are emitted in the aerogel itself or in the diffusing box. The contribution to the background from delta rays is estimated to be $\simeq 0.12$ p.e. The effect of secondary hadronic interaction 
is negligible. A dedicated proton run at $1 \mathrm{GeV} / \mathrm{c}$ where delta rays in aerogel are below Cherenkov thtreshold and protons in PTFE walls are closed to the Cherenkov threshold, shows that the remaining background attributed to scintillation is $\leq 0.14$ p.e. From these observations we conclude that the main contribution to the background at $5 \mathrm{GeV} / \mathrm{c}$ is coming from the Cherenkov light emitted in the PTFE wrapping ( $\simeq 0.6 \mathrm{p} . \mathrm{e})$.

Figure 8 shows the proton contamination: $(\mathcal{P}(p \rightarrow \pi))$ and the pion inefficiency: $(\mathcal{P}(\pi \rightarrow p))$ as a function of the cut we apply to decide if an event corresponds to a pion or a proton. $\mathcal{P}(p \rightarrow \pi)$ is the probability for a proton to give light in the counter and to be identified as a pion and $\mathcal{P}(\pi \rightarrow p)$ is the probability for a pion, not to give enough light in the counter and to be identified as a proton. For convenience, the point for which those two probabilities are equal is considered as a figure of merit of our detector and permits us to compare different configurations. This analysis has been repeated for 2 runs

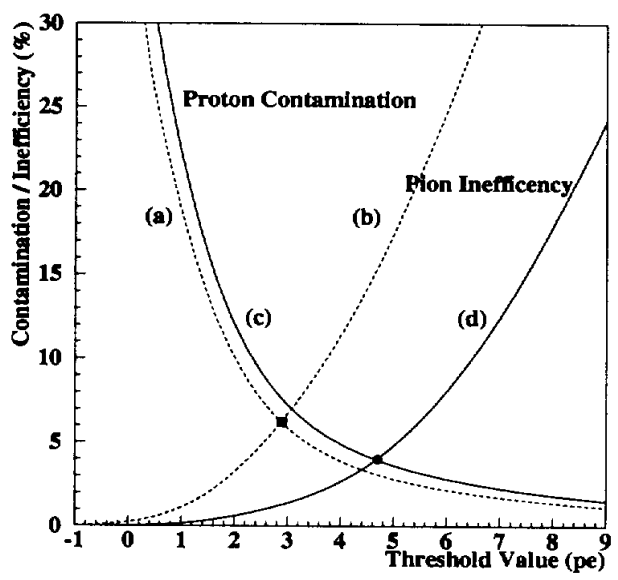

Fig. 8. $\mathcal{P}(\pi \rightarrow P)$ and $\mathcal{P}(P \rightarrow \pi)$ as a function of the threshold in photo-electrons. (a),(b) are for an average number of photo-electrons equal to 10.0 for $\beta=1$ particles and (c),(d) are for an average number of 13.8

with a different average number of photo-electrons (Npe) for $\beta=1$ incident particles: figure 8 (a),(b) shows these probabilities for $\mathrm{Npe}=10.0$ corresponding to the diffusing box without PMP, the equality of the probabilities occurs for a threshold set to 2.9 pe, the misidentification is then equal to $6.2 \%$. Figure 8 (c),(d) shows the same for Npe=13.8 corresponding to the box with PMP, the equality of the probabilities occurs for a threshold equal to 4.7 pe, the misidentification is then equal to $4.0 \%$. 


\section{Monte Carlo simulation}

\section{Description}

Using GEANT 3.21 package [13], we have simulated the low and high index aerogel cells with the geometries of the test beam setup. The Cherenkov photons are tracked with standard GEANT subroutines, but we had to introduce in the aerogel the Rayleigh scattering phenomenon with an associated $\lambda^{4}$ dependent interaction length; this latter is typically few centimeters at 500 $\mathrm{nm}$. Figure 9 shows the simulated Cherenkov photons trajectories following the crossing of a kaon of $4.1 \mathrm{GeV} / \mathrm{c}$ in low index aerogel $(\mathrm{n}=1.008)$.

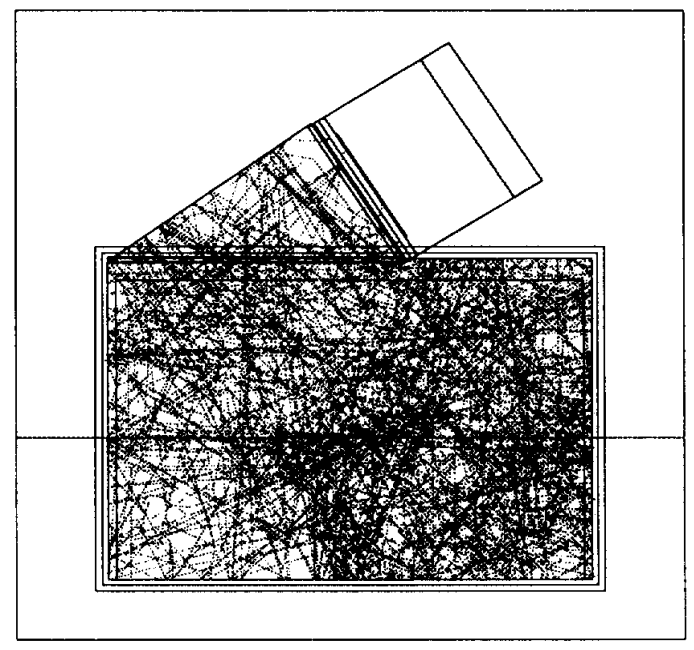

Fig. 9. Simulated scattered photons produced by a kaon of $4.1 \mathrm{GeV} / \mathrm{c}$ in low index aerogel $(n=1.008)$.

Another improvement concerned the photon reflection on the PTFE walls. Three components exist: the diffuse emission according to the Lambert's law, the specular reflection and the absorption. Measurements [10] have been made to determine for various incident angles, the relative proportion of diffuse to the specular reflection. Specular reflection becomes important for incident angles greater than 70 degrees. For lower incident angles, where diffused reflexion dominates, the preferential direction of reflected photons is the normal to the PTFE wall.

We have introduced in the simulation the photo-cathode efficiency for each fine mesh phototube used during the test beam. For the 2 in. phototube we had specific measurement of this quantity but for the $1.5 \mathrm{in}$. one we had only typical values from the constructor 
In figure 10 , is shown the evolution of Cherenkov photons wavelength distribution between successive reflections on the PTFE walls. In figure 11 are

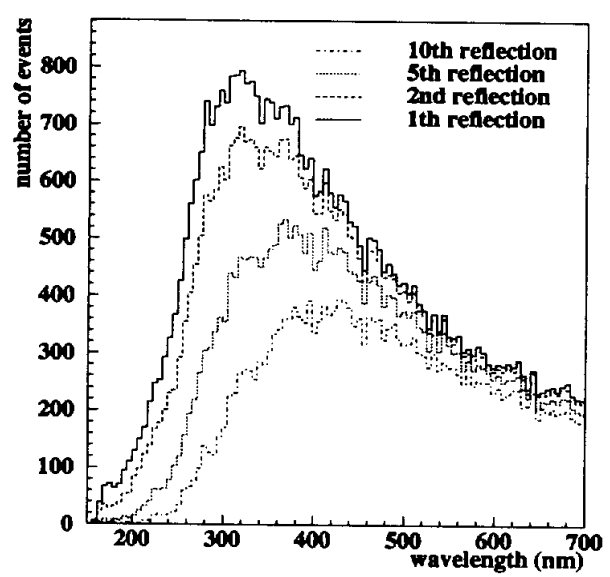

Fig. 10. $\lambda$ distributions of photons after the first, the second the fifth and the tenth reflection on the PTFE walls. Low index aerogel is inside the cell.

given the wavelength distributions of photons reaching the photo-cathode versus the vertical position, of an incident pion. As shown in the figures 10 and 11 , the UV photons are, highly and quickly absorbed in the aerogel. Shifting the wavelength towards blue will increase the number of photons that will reach the photo-cathode and will produce a more uniform response along the vertical direction. We have simulated the effect of the PMP 420 for the low index aerogel.

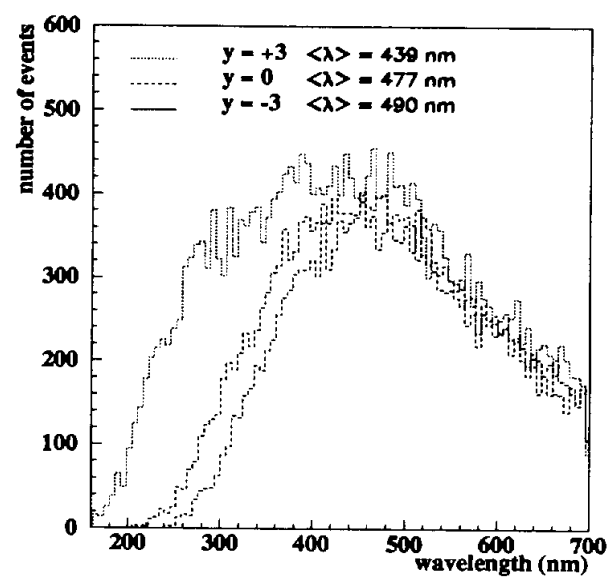

Fig. 11. $\lambda$ distributions of photons reaching the photo-cathode versus the pion incident position along the vertical direction $y$. Low index aerogel is inside the cell. 
The number of photo-electrons as well as the shape of the response along the vertical direction are strongly dependent on the photo-cathode efficiency $\varepsilon$, the absorption length $L_{a}$, the scattering length $L_{s}$ in the aerogel, and the absorption on PTFE walls $A$ [14]. These quantities all depend on the Cherenkov photons wavelength $\lambda$.

We then studied separatly the influence of each physical variable $\varepsilon, A, L_{a}$ and $L_{s}$ on the number of photo-electron, despite the fact that these effects are correlated.

- Effect of $\varepsilon(\lambda)$ variation : Two types of photo-cathode were considered : UV glass with normal efficiency (black squares in figure 12.a) and normal glass with high quantum efficiency (black triangles in figure 12.a). In this study, walls absorption, absorption and scattering length in aerogel are fixed to the values measured by the Novosibirsk group [8] (black squares in fig. 12.a, 14.a and 15.a). In figure 12.b we note that the number of photo-electrons as well as the shape of the vertical response are dependent on $\varepsilon$, the photocathode acting as a filter.
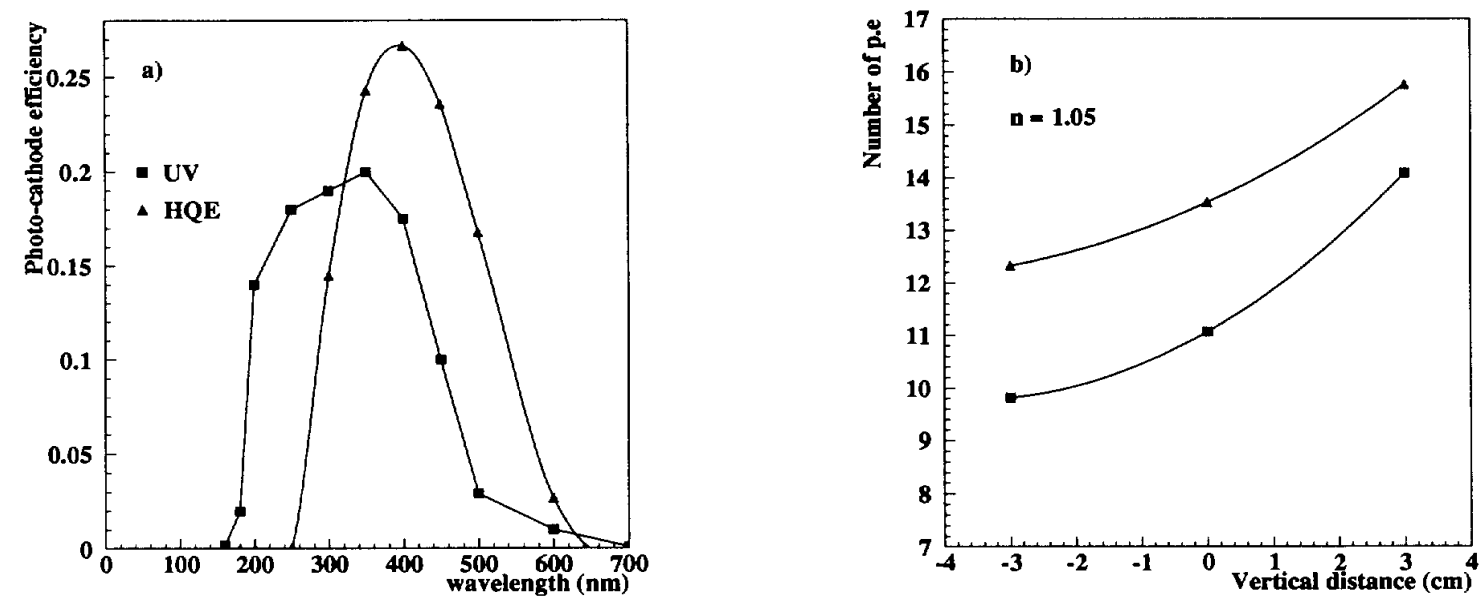

Fig. 12. Influence of photo-cathode efficiency.

a) Efficiency $\varepsilon(\lambda)$. Two types of photo-cathode are used: UV glass and normal efficiency (black squares) and normal glass and high quantum efficiency (black triangles).

b) Number of photo-electrons for the two types of photo-cathode.

- Effect of $A(\lambda)$ variation: As starting values, we used absorption coefficients $A(\lambda)$ of PTFE, measured from the Novosibirsk group and extrapolated to short wavelengths. Absorption and scattering length in aerogel are fixed respectively to black squares in fig. 14.a and fig. 15.a. Figure 13 shows the influence of the reflectivity on the number of photo-electrons. 

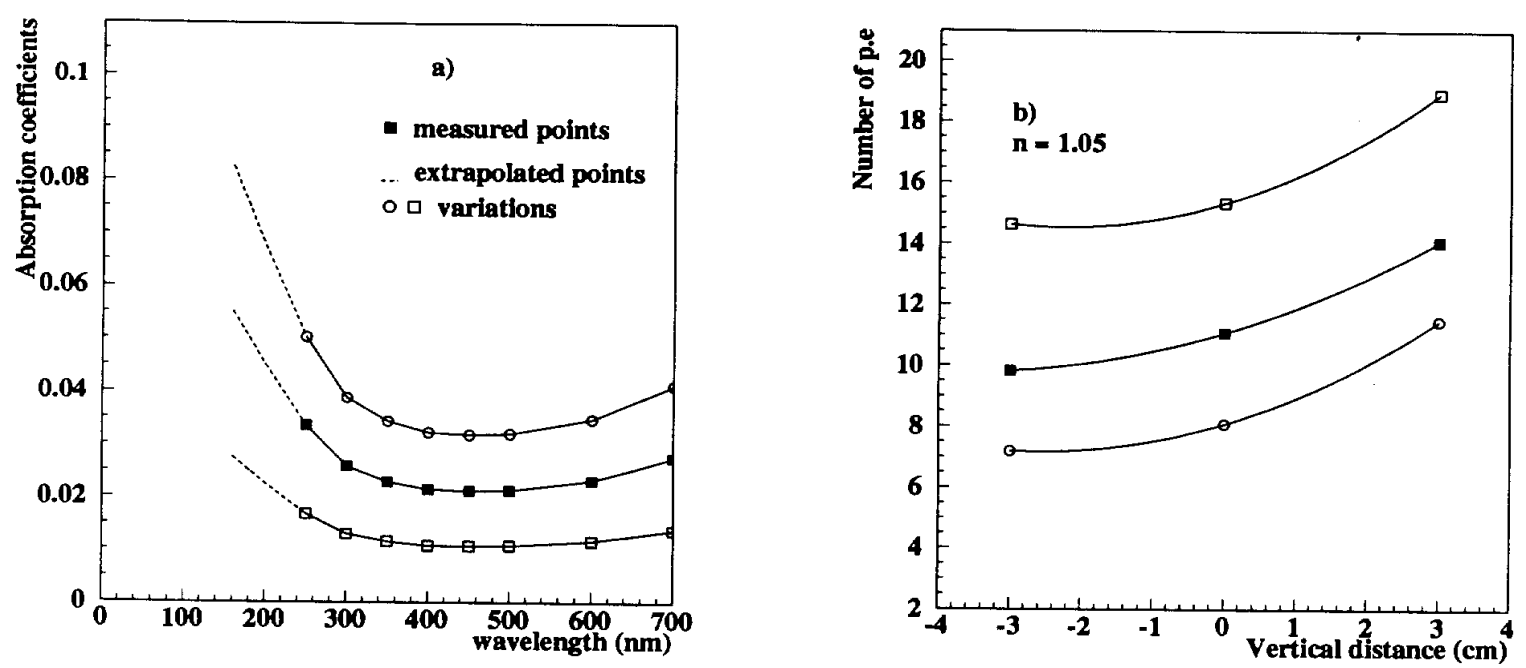

Fig. 13. Influence of walls absorption.

a) Absorption coefficient $A(\lambda)$, of the PTFE wall. The black squares are the measured points. White squares and circles correspond to variations.

b) Corresponding number of photo-electrons as a function of the vertical position.
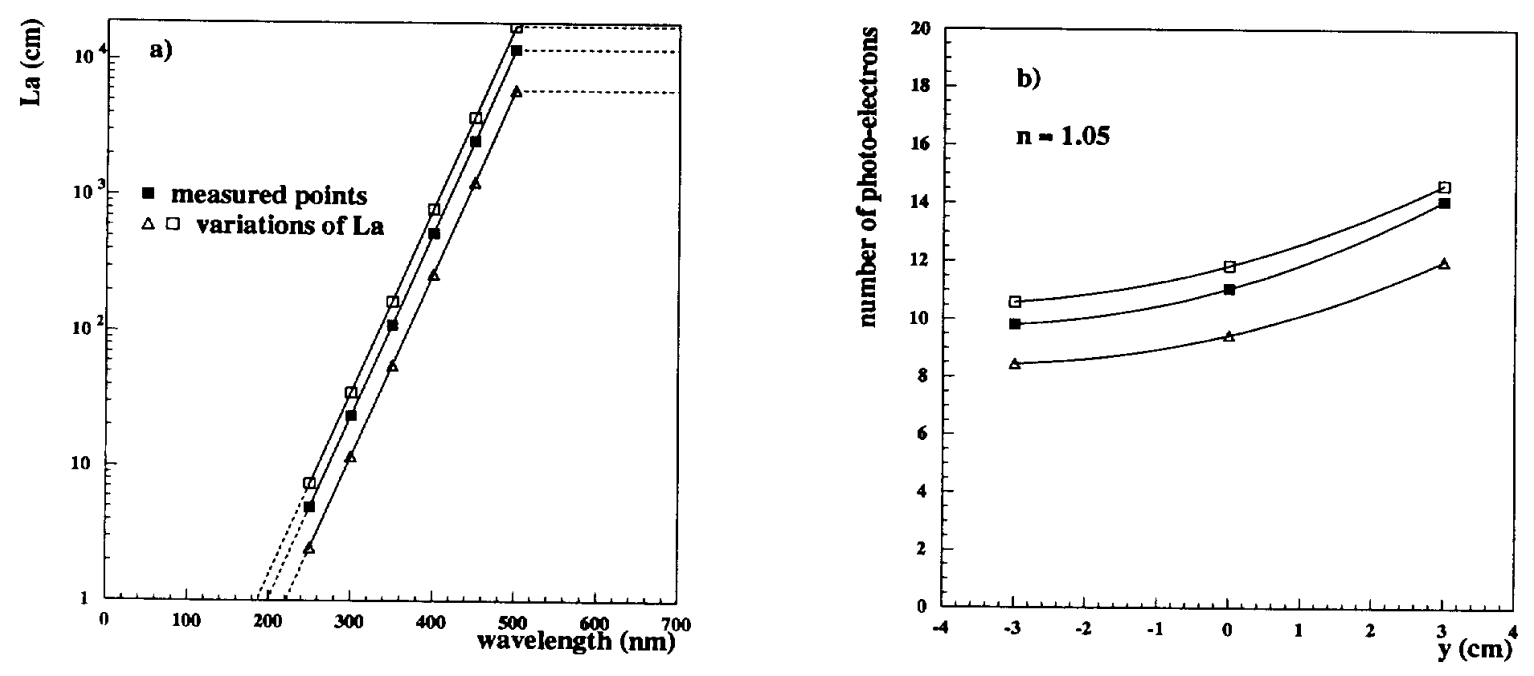

Fig. 14. Influence of aerogel absorption length.

a) Aerogel absorption length $L_{a}(\lambda)$. The black squares are the measured points. White triangles, squares and circles correspond to variations.

b) Corresponding number of photo-electrons as a function of the vertical position.

- Effect of $L_{a}(\lambda)$ variation: Starting from the measurement of the absorption length $L_{a}(\lambda)[8]$ (black squares in figure 14.a), we modified slightly the $L_{a}(\lambda)$ shape. The scattering length is taken equal to $4 \mathrm{~cm}$ at $436 \mathrm{~nm}$. In figure 14.b we observe that if the number of photo-electrons is sensitive to the 
absorption length, the shape of the response along the vertical direction is roughly conserved (for our homothetical variation).

- Effect of $L_{s}(\lambda)$ variation: We studied, in the same way, the influence of the variation of the scattering length in aerogel by multiplying the measured points $L_{s}(\lambda)$ (black squares on the figure 15.a) by different scale factors. The absorption length in aerogel is fixed to black squares in figure 14.a. We see on the figure 15.b that the decrease of the scattering length induces a larger dynamic on the vertical response.
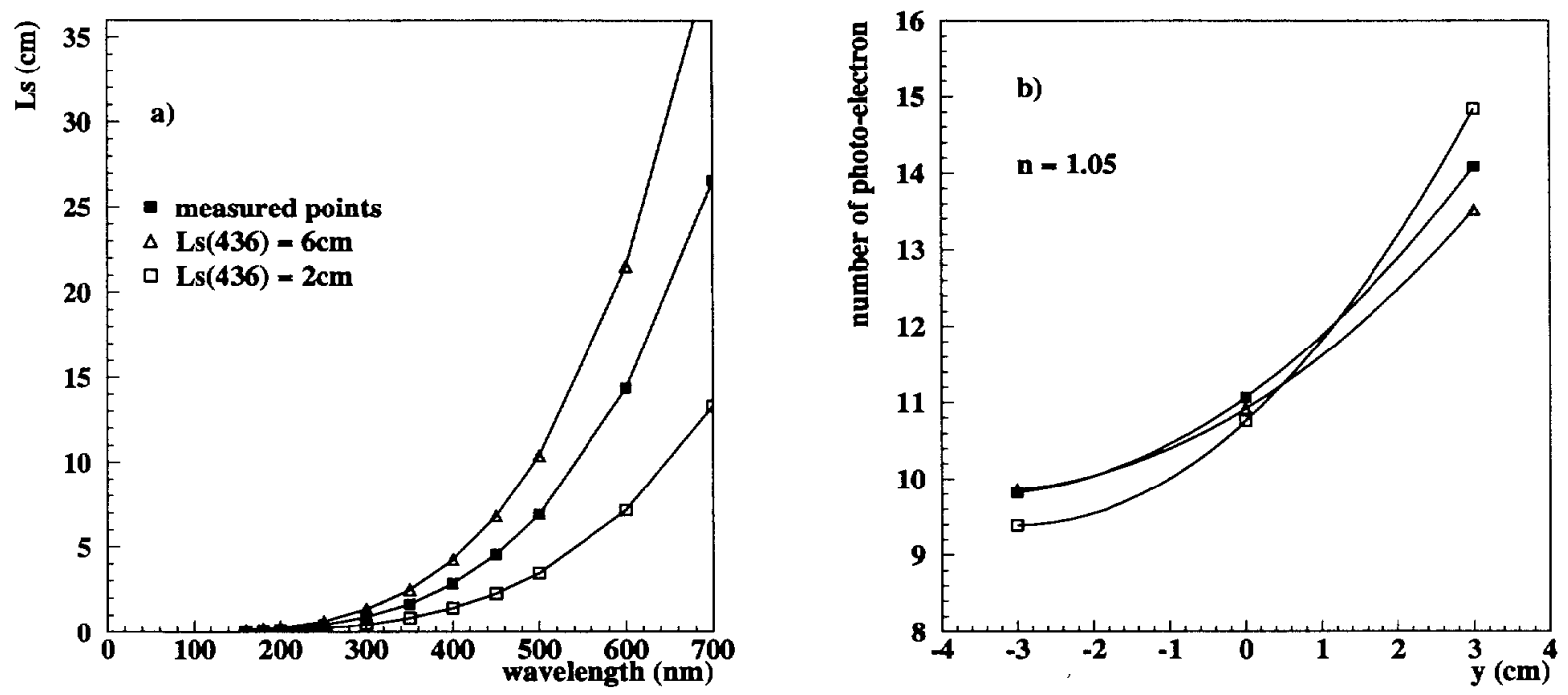

Fig. 15. Influence of aerogel scattering length.

a) Aerogel scattering length $L_{s}(\lambda)$. The black squares are the measured points. white squares and white triangles correspond respectively to $L_{s}(436)=2 \mathrm{~cm}$ and $L_{s}(436)=6 \mathrm{~cm}$.

b)Corresponding number of photo-electrons as a function of the vertical position.

- Effect of $L_{a}$ using PMP: The PMP-420 wavelength shifter shifts light from around $320 \mathrm{~nm}$ to $420 \mathrm{~nm}$. Thus the increase of photo-electrons detected using PMP-420 on the cell walls higly depends on the aerogel absorption length beetween 250 and $450 \mathrm{~nm}$. As shown in figure 16, in order to benefit from a noticeable increase of the photocatode yield, the aerogel must be quite transparent at least down to $350 \mathrm{~nm}$. Above this last wavelength, the increase of photoelectrons is governed by the photo-cathode sensitivity.

\section{Comparison between data and simulation}

- High index aerogel: It is very difficult to measure correctly the aerogel absorption length independently of the scattering length. However, measurements [8] have been made by the Novosibirsk group (black squares on 

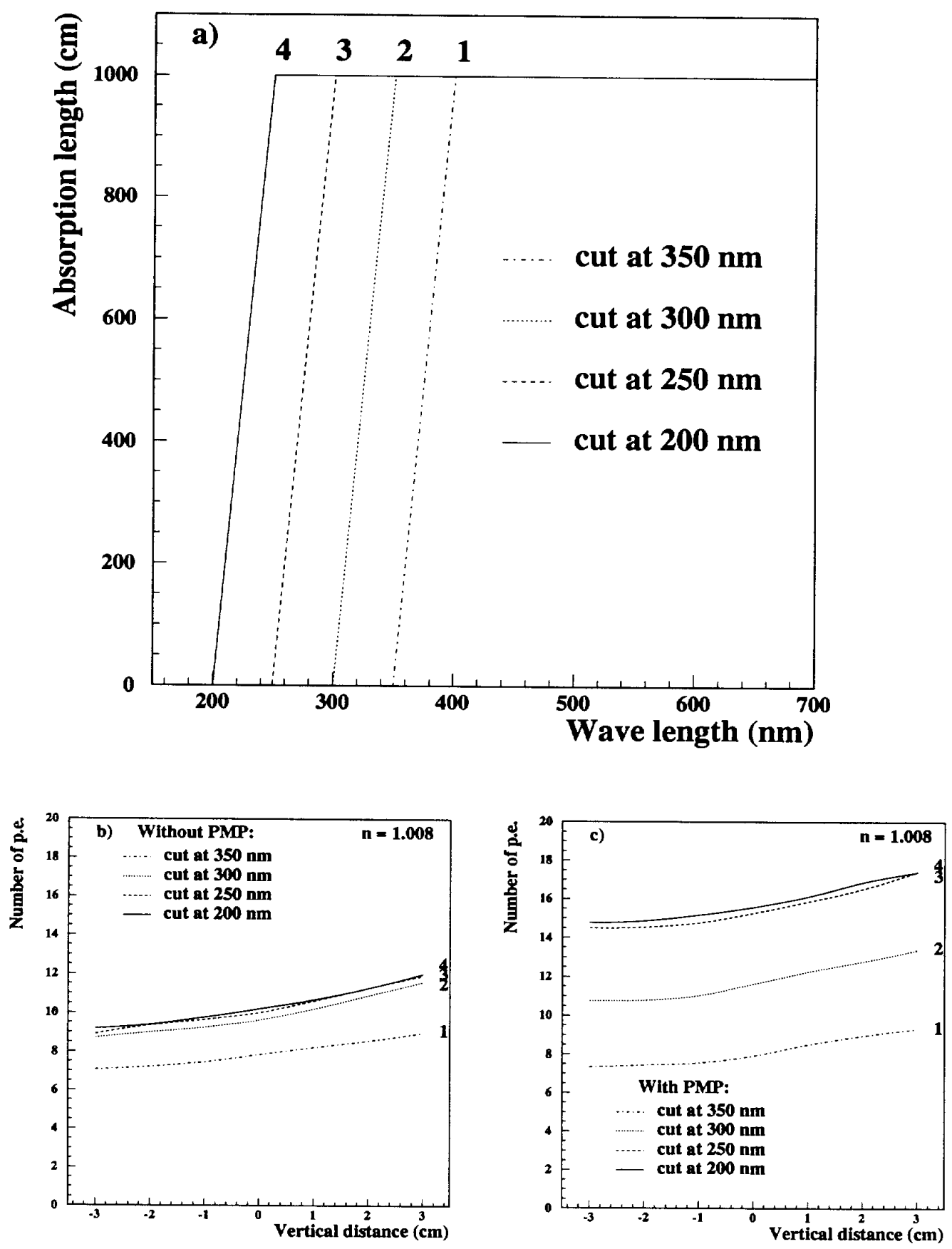

Fig. 16. Influence of aerogel absorption length with PMP.

a) Simple aerogel absorption length at different thresholds.

b) Number of photo-electrons for various absorption threshold, without PMP.

c) Same as b), with PMP. 


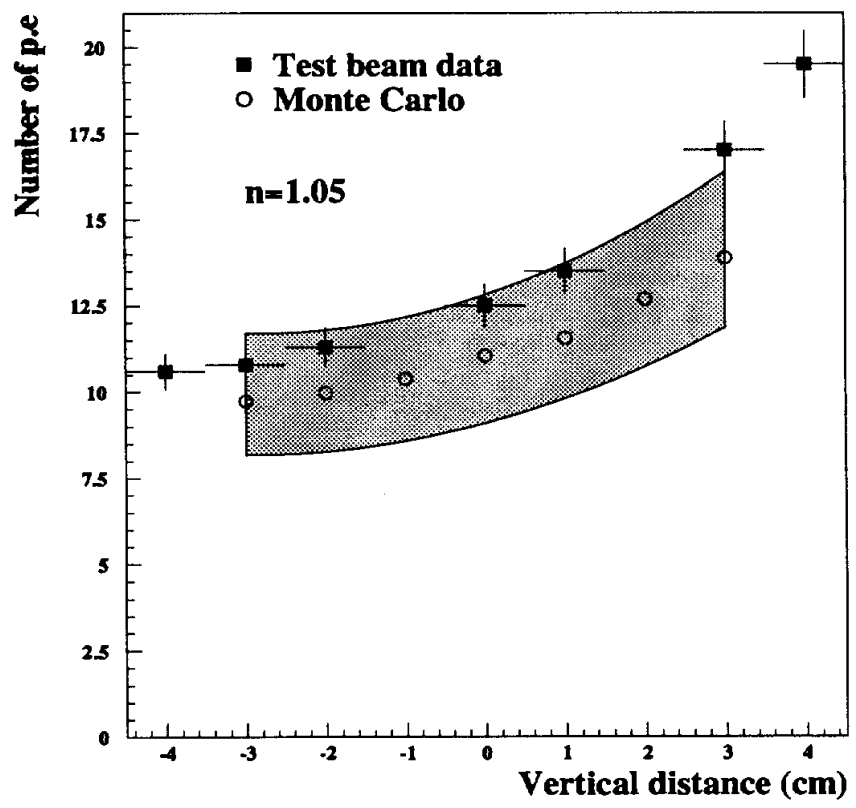

Fig. 17. Comparison data Monte Carlo for the high index aerogel $(n=1.05)$. The region in grey corresponds to the uncertainty on the number of p.e. coming from the error on the parameters: $L_{a}(\lambda), A(\lambda)$ and $\varepsilon(\lambda)$. The error on the points are strongly correlated together

figures 14.a and 15.a). We introduced these values in the simulation and for PTFE absorption, we took the measured points (black squares on figure 13.a). On the figure 17, we obtain a good agreement between data and simulation for the number of photo-electrons but the shape of the vertical response is not well reproduced. However, it has been possible to reproduce this latter by choosing a scattering length of $2 \mathrm{~cm}$ at $436 \mathrm{~nm}$. The domain in which the number of photo-electron is allowed to vary (in grey on figure 17) has been computed by summing in quadrature the measurement uncertainties on the optical parameters: $L_{a}(\lambda), A(\lambda)$ and $\varepsilon(\lambda)$. The main contribution to the total error comes from the quantum efficiency $\varepsilon(\lambda)$ for which we had no specific measurement.

- Low index aerogel: Aerogel absorption and scattering length measurements are not yet available for this index. However the comparison of the data with and without PMP, gives us a constraint on aerogel absorption length. Therefore, keeping measurements of the PTFE absorption, we obtained a good agreement for an absorption length of $1000 \mathrm{~m}$ down to 400 $\mathrm{nm}$ and then reaching 0 at $200 \mathrm{~nm}$, and a scattering length of $4 \mathrm{~cm}$ at 436 $\mathrm{nm}$. The figure 18 shows the comparison between data and Monte Carlo, obtained with these rough parameters. We must point out that the correlations between the effect of the different parameters and the low speed of 
the Monte-Carlo program make difficult any optimisation.

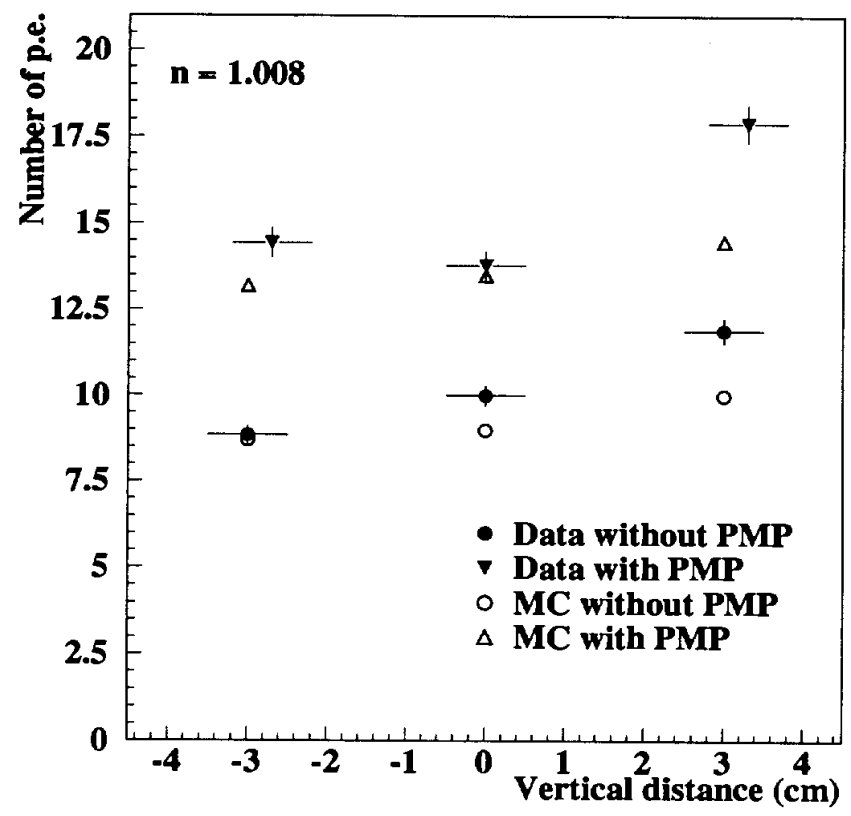

Fig. 18. Comparison data Monte Carlo for the low index aerogel $(n=1.008)$.

Other results from simulation

In order to understand the photon collection efficiency, we extract from the simulation the following results:

- Mean free path for photons reaching the photo-cathode, using fitted aerogel scattering and absorption length:

\begin{tabular}{||c||c|c||}
\hline & high index & low index \\
\hline \hline mean free path $(\mathrm{cm})$ & 181 & 245 \\
\hline Nb of reflections on the walls & 30 & 29 \\
\hline Nb of Rayleigh diffusions & 81 & 110 \\
\hline
\end{tabular}

- Number of photo-electrons versus photo-cathode diameter: this number is linear with the photo-cathode diameter and not proportionnal to its surface as can been seen on the figure 19 . 


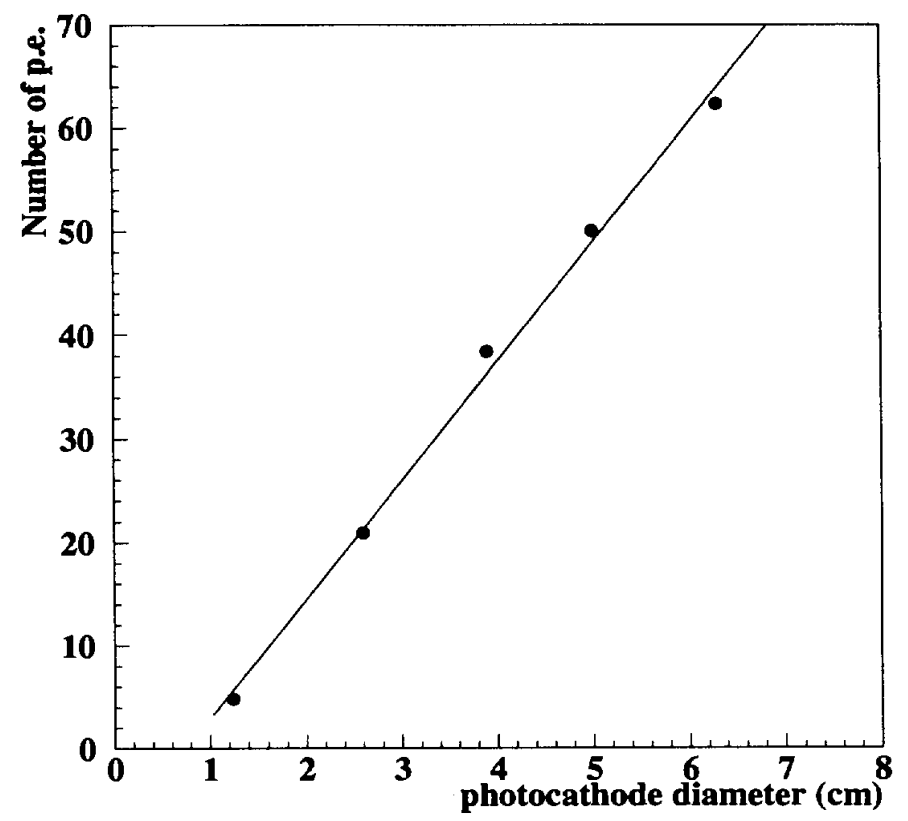

Fig. 19. Number of photoelectrons versus photo-cathode diameter.

\section{Conclusion}

We have performed a test on an aerogel threshold counter equipped with two cells containing a low (1.008) and a high (1.05) index aerogel. Both cells were read out by fine mesh Hamamatsu photo-multipliers. The observed average number of photo-electrons for $\beta=1$ particles is greater than 10 for the high index, greater than 8.7 for the low index and PTFE wrapping for the reflective walls. The addition of a further layer of PTFE impregnated with PMP-420 improves the performances of the low index cell, and permits to observe more than 13 photo-electrons for every impact point position of the incident particle.

The study of background induced by below threshold particles shows that the low index aerogel prototype allows to reach a pion selection efficiency equal to $96 \%$ for a proton contamination as low as $4 \%$.

A complete Monte-Carlo study of the optical parameters influence has been performed.

We conclude that silica aerogel read out by fine mesh photo-multiplier can be used to build a powerful and compact particle identification device in the momentum region $0.4-4.3 \mathrm{GeV} / \mathrm{c}$. 


\section{Acknowledgments}

We would like to thank Didier Puertolas from CERN for fruitful discussions on the use of PMP wavelength shifter.

We acknowledge the CERN-PS accelerator team for its excellent work and help during the beam test.

We are greatly grateful to P. Tsou and L. Hrubesh for their R\&D effort of producing high quality aerogel blocks and their continuous support since 1992.

\section{References}

[1] D. Boutigny et al., BaBar Technical Design Report, SLAC-R-95-457, March 1995.

[2] G. Eigen, D.G. Hitlin and J. Oyang, "Silica aerogel Cherenkov detectors for particle identification", CALT-68-1829, October 1992.

[3] "B factories; The state of the art in accelerators, detectors, and physics", SLAC400, November 1992.

[4] "Status report on the design of a detector for the study of CP violation at PEPII at SLAC", SLAC-419, June 1993.

[5] J. Oyang, "Performance of an aerogel particle identification system", BaBar Note 137.

[6] X. Shi,"Geant simulation of an aerogel particle id system", BaBar Note 142.

[7] BaBar aerogel group, D. Boutigny et al, "Performance of an aerogel threshold particle identification detector read out by Hybrid Photon Detectors", BaBar Note 291.

[8] BaBar aerogel group, K. Arisaka et al, "Performance of a 4-Layer Prototype Aerogel Counter" BaBar Note 290.,

[9] D. Boutigny, talk presented at the VI International conference on instrumentation for experiments at $e^{+} e^{-}$colliders.

published as: K. Arisaka et al. "Recent results on prototype aerogel threshold counters for particle identification in the region:0.5-4.3 GeV/c" LAPP preprint LAPP-EXP-96.06.

http://lapphp0.in2p3.fr/preplapp/psexp/lappexp9606.ps.gz

[10] Padova group private communication

[11] C. D'Ambrosio et al., "Organic scintillators with large Stokes shifts dissolved in polystyrene", NIM A 307 (1991) 430-435 and references therein. 
[12] R. Enomoto et al., "Feasibility Study of Single-Photon Counting Using a Finemesh Photo-tube for an aerogel Readout", NIM. A 332 (1993) 129-133.

[13] R. Brun et al., GEANT 3.15 CERN DD/78-2

[14] A.R. Buzykaev et al. "Project of aerogel Cherenkov counters for KEDR" Talk presented at the VI International Conference on Instrumentation for experiment at $e^{+} e^{-}$colliders, Novosibirsk, March 1996 
. 